\title{
EPSAC Predictive Control Applied to Muscle Relaxant Administration
}

\author{
Ramona Hodrea*, Ioan Nascu*, Robin de Keyser**, Clara M. Ionescu** \\ *Technical University of Cluj-Napoca, Automation Department, Observatorului 2, Cluj-Napoca, Romania \\ Email: ramona.hodrea@aut.utcluj.ro \\ **Ghent University, Department of Electrical energy, Systems and Automation, Faculty of Engineering \\ Technologiepark 913, Gent B9052 Belgium
}

\begin{abstract}
An Extended Prediction Self-Adaptive Control (EPSAC) strategy is applied to the rocuronium administration, a neuromuscular blocking drug used in anaesthesia to facilitate endotracheal intubation and to provide skeletal muscle relaxation during surgery. A safe anaesthesia should provide comfort to the patient and best possible working conditions for the surgeon. The muscle relaxant plays an important role and an adequate muscle relaxation, which allows efficient and safe surgery, can be provided using automated control. Moreover, closed-loop control may reduce anesthetist workload and achieve better regulation of muscle relaxation than manual administration, calculating the dose required for each patient and avoiding in this way overdosing. Predictive control requires prior knowledge of system dynamics, therefore a mathematical model is required. A three-compartment model for rocuronium is presented. Several parameter sets are used to simulate different patients and control performance is discussed.
\end{abstract}

Keywords: Predictive control, anaesthesia, compartmental model, drug dosing control

\section{INTRODUCTION}

Muscle relaxants are used during surgeries and in intensive care units to facilitate management of ventilation, to control intracranial hypertension, to reduce oxygen consumption, to eliminate shivering, and to provide immobility for certain diagnostic studies. Patients receiving muscle relaxants have critical illness, therefore it is important to select the appropriate drug. The rocuronium properties make it suitable for continuous administration (McCoy et al. 1996) and for target controlled infusions (TCI). The duration of action is similar to that of vecuronium, but it has more rapid onset of action (Foldes et al. 1991). It is classified as an intermediateduration non-depolarizing neuromuscular blocking agent.

Pharmacological modeling has been used to describe the metabolism of muscle relaxants for more than three decades (Farenc et al. 2001; Wierda et al. 1990; Vermeyen, Hoffman and Saldien 2003). Compartmental models are formulated on the basis of the minimal number of compartments that adequately fits observed data. This type of model is widely used in different control strategies. Using an automatic control system to maintain skeletal muscle relaxation increases the accuracy of the desired neuromuscular block and minimizes the administered drug at the same time. Furthermore, more appropriate level of relaxation corresponding to the clinical requirement can be targeted as the observation interval is considerably reduced. Closed-loop muscle relaxation control may also play a role in the detection of insufficient level of sedation. Giving a too high dose of muscle relaxant to prevent movement could increase the risk of not detecting insufficient sedation. Closed-loop control avoids complete paralysis, allowing in some cases patient movement to indicate patient responsiveness.

Automatic feedback control of skeletal muscle relaxation has been addressed by several research groups, mainly because syringe pumps can be easily controlled by computer systems and more importantly a direct and reliable measure of effect is available. A variety of control strategies can be found in the literature for muscle relaxants, ranging from on-off, PID controllers (De Vries, Ros \& Booij 1986) to model based and adaptive controllers (Kansanaho \& Olkkola 1996). Due to the fact that PID controllers cannot anticipate the response of the patient and do not have any knowledge of the drug metabolism, stability problems are present. Predictive control has some advantages: it is robust against variable and unknown time-delay, over parameterization of system models, and has good disturbance rejection properties.

This paper presents an Extended Prediction Self-Adaptive Control (EPSAC) strategy implemented for the administration of rocuronium during general anaesthesia. An overview of the pharmacokinetic and pharmacodynamic patient model is given in the next section. The NEPSAC (Nonlinear EPSAC) approach to MPC is presented in section 3. Simulation results of the closed-loop NEPSAC predictive control strategy when different parameter sets are used to 
simulate the patients are discussed in section 4. The conclusions are summarized in a final section and future step is suggested.

\section{PATIENT MODEL}

The pharmacological modeling comprises two main categories known as pharmacokinetics (PK) and pharmacodynamics (PD). The former category studies the relationship between drug dose and drug concentration existing in the blood plasma, while the latter deals with the effect produced by the drug.

The PK model considered in this paper consists of three compartments. Two peripheral compartments are arranged around a central compartment. After the drug injection, the plasma concentration of rocuronium declines rapidly in exponential phases corresponding to distribution to peripheral compartments and elimination. To identify the drug effect, a $4^{\text {th }}$ compartment known as the effect compartment has been introduced to the rocuronium kinetics (Fig.1).

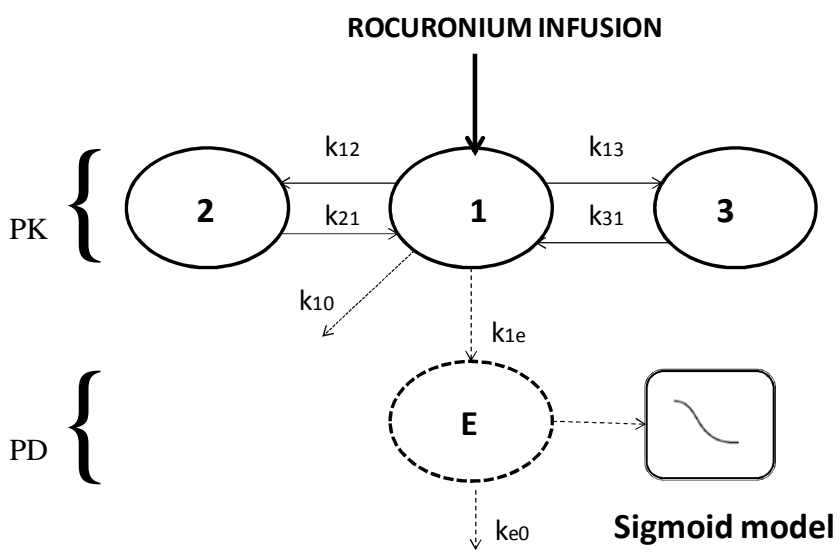

Fig.1. PK-PD compartmental model

The pharmacokinetics described by the three-compartment model is expressed by the following equations:

$$
\begin{aligned}
\dot{x}_{1}(t) & =-\left[k_{10}+k_{12}+k_{13}\right] \cdot x_{1}(t)+k_{21} \cdot x_{2}(t) \\
& +k_{31} \cdot x_{3}(t)+\frac{u(t)}{\text { weight } \cdot V_{c}} \\
\dot{x}_{2}(t) & =k_{12} \cdot x_{1}(t)-k_{21} \cdot x_{2}(t) \\
\dot{x}_{3}(t) & =k_{13} \cdot x_{1}(t)-k_{31} \cdot x_{3}(t)
\end{aligned}
$$

where $x_{1}, x_{2}, x_{3}$ denote the concentration of drug $[\mathrm{mg} / \mathrm{ml}]$ in the central (compartment 1) and peripheral compartments (compartments 2 and 3). The parameters $k_{j i}$, for $i \neq j$, denote the rate constants for equilibration between compartments. $V_{c}$ is the volume of distribution per $\mathrm{kg}$ of body weight in the central compartment. The constant $k_{10}$ is the rate constant for the processes that irreversibly remove drug from the central and peripheral compartments, and $u(t)$ $[\mathrm{mg} / \mathrm{s}]$ is the infusion rate of the rocuronium into the central compartment.

Inter-compartmental constant rate values for PK model found in the literature are presented below. The values are obtained based on studies on different groups of patients. Mean values for the age and weight of these patients are given as well.

Table 1. Pharmacokinetic parameter sets for rocuronium as found in the literature: Set 1 (Alvarez-Gomez et al. 1994); Set 2 (Wierda et al. 1991); Set 3 (Szenohradszky et al. 1992); Set 4 (Cooper et al. 1993)

\begin{tabular}{lllll}
\hline & Set 1 & Set 2 & Set 3 & Set 4 \\
\hline $\mathbf{V}_{\mathbf{C}}\left(\mathrm{ml} \mathrm{kg}^{-1}\right)$ & 57 & 45 & 77 & 69 \\
\hline $\mathbf{k}_{\mathbf{1 0}}\left(\mathrm{min}^{-1}\right)$ & 0.0952 & 0.1 & 0.0375 & 0.119 \\
\hline $\mathbf{k}_{\mathbf{1 2}}\left(\mathrm{min}^{-1}\right)$ & 0.2807 & 0.21 & 0.1142 & 0.259 \\
\hline $\mathbf{k}_{\mathbf{2 1}}\left(\mathrm{min}^{-1}\right)$ & 0.2149 & 0.13 & 0.1758 & 0.163 \\
\hline $\mathbf{k}_{\mathbf{1 3}}\left(\mathrm{min}^{-1}\right)$ & 0.0322 & 0.028 & 0.0196 & 0.06 \\
\hline $\mathbf{k}_{\mathbf{3 1}}\left(\mathrm{min}^{-1}\right)$ & 0.0166 & 0.01 & 0.0189 & 0.012 \\
\hline weight $(\mathrm{kg})$ & 70 & 68 & 78 & 69 \\
\hline age $(\mathrm{yr})$ & 30 & 51 & 44 & 46 \\
\hline
\end{tabular}

Regarding the pharmacodynamic (PD) model, an additional hypothetical effect compartment was proposed to represent the lag between drug plasma concentration and drug response. The effect compartment receives drug from the central compartment by a first-order process, but it is assumed that the quantity of drug is so small that it does not affect the pharmacokinetic model. The input to the effect site compartment is expressed by a first-order rate constant, $k_{l e}$. The output is also expressed by a first-order rate constant, $k_{e} 0$. This effect site compartment is represented by the following equation:

$$
\dot{x}_{e}(t)=-k_{e 0} \cdot x_{e}(t)+k_{1 e} \cdot x_{1}(t)
$$

where $k_{e 0}=k_{1 e}=0.168\left[\mathrm{~min}^{-1}\right]$ and $\quad x_{e} \quad$ is the drug concentration in the effect compartment (Sheiner et al. 1979). The effect compartment is defined as a negligibly small compartment connected to the central compartment. Knowing $k_{e 0}$, the apparent concentration in the effect compartment can be calculated since $k_{e 0}$ will precisely characterize the temporal effects of equilibration between the plasma concentration and the corresponding drug effect.

The level of neuromuscular block can be related to the drug concentration in the effect compartment $x_{e}$ by the following sigmoid equation:

$$
E(t)=E_{0}-E_{\max } \frac{x_{e}^{\gamma}(t)}{x_{e}^{\gamma}(t)+x_{e, 50}^{\gamma}}
$$

where $E(t)$ is the degree of muscle relaxation, $E_{\max }$ denotes the maximum effect achieved by the muscle relaxant, $x_{e, 50}$ is the drug concentration at half maximal effect and represents the patient sensitivity to the drug, and $\gamma$ determines the steepness of the curve. The following values are taken from (Vermeyen et. al. 2003): $E_{\max }=100 ; E_{0}=100 ; \gamma=4.79$;

$$
x_{e, 50}=0.823[\mu \mathrm{g} / \mathrm{ml}] \text {. }
$$


In clinical practice the degree of muscle relaxation or the level of neuromuscular block is measured by assessing responses to peripheral nerve stimulations, typically on the ulnar nerve. The usual method to assess degree of relaxation is based on a train-of-four (TOF) stimulation and the respective measurement of response. To perform a train-offour (TOF) pattern of nerve stimulation, 4 supramaximal electrical stimuli are applied over the course of a 1.5 to 2.0 seconds period (frequency of $2 \mathrm{~Hz}$ ). Each stimulus causes a muscle contraction, and the "fade" of response to this stimulus is measured. Dividing the level of the fourth response $\left(r_{4}\right)$ by the level of the first response $\left(r_{1}\right)$ provides the TOF ratio:

$$
\text { TOF }=100 \cdot \frac{r_{4}}{r_{1}}
$$

TOF ratio can be analytically calculated using relation (3).

\section{CONTROL ALGORITHM}

EPSAC strategy, as described in detail in (De Keyser 2003), is based on a generic process model:

$$
y(t)=x(t)+n(t)
$$

The signal $x(t)$ is the model output resulting from the model (3) input $u(t)$. The disturbance $n(t)$ includes the effects in the measured output $y(t)$ which do not come from the model input $u(t)$ via the available model. These non-measurable disturbances have a stochastic character with non-zero average value, which can be modelled by a coloured noise process:

$$
n(t)=\left[C\left(q^{-1}\right) / D\left(q^{-1}\right)\right] \cdot e(t)
$$

with: $e(t)$ - uncorrelated (white) noise with zero mean value; $C\left(q^{-1}\right)$ and $D\left(q^{-1}\right)$ - monic polynomials in the backward shift operator $q^{-1}$ of orders $n_{c}$ and $n_{d}$. The disturbance filter $C\left(q^{-1}\right) / D\left(q^{-1}\right)$ is considered to be a design filter (De Keyser $\&$ Ionescu 2003) and it plays an important role in MPC.

\subsection{Prediction Algorithm}

The model output $x(t)$ represents the effect of the control input $u(t)$ on the process output $y(t)$ and is also a nonmeasurable signal, and the relationship between $u(t)$ and $x(t)$ is given by the generic dynamic system model:

$$
x(t)=f[x(t-1), x(t-2), \cdots, u(t-1), u(t-2), \cdots \sqsubset
$$

The fundamental step in MPC methodology consists in prediction of the process output $y(t+k)$ at time instant $t$, indicated by $\left\{y(t+k / t), k=1 . . N_{2}\right\}$, over the prediction horizon $N_{2}$, and based on:

- the measurements available at sampling instant $t$ :

$$
\{y(t), y(t-1), \cdots, u(t-1), u(t-2), \cdots\} \text {; }
$$

- The future values of the input signal (postulated at time $t):\{u(t \mid t), u(t+1 \mid t), \cdots\}$.

Using the generic process model (5), the predicted values of the output are:

$$
y(t+k \mid t)=x(t+k \mid t)+n(t+k \mid t)
$$

Prediction of $x(t+k \mid t)$ and of $n(t+k \mid t)$ can be done respectively by recursion of the process model (7) and by using filtering techniques on the noise model (6) (De Keyser, 2003).

\subsection{Control Algorithm}

In EPSAC for linear models, the future response is then considered as being the cumulative result of two effects:

$$
y(t+k \mid t)=y_{\text {base }}(t+k \mid t)+y_{\text {optimize }}(t+k \mid t)
$$

The two contributions have the following origins:

$y_{\text {base }}(t+k \mid t)$ :

- $\quad$ effect of past control $\{u(t-1), u(t-2), \ldots\} \quad$ (initial conditions at time $t$ );

- effect of a base future control scenario, called $u_{\text {base }}(t+k \mid t), \quad k \geq 0$, which is defined a priori (De Keyser 2003); for linear systems the choice is irrelevant, a simple choice being $\left\{u_{\text {base }}(t+k \mid t) \equiv 0, \quad k \geq 0\right\}$;

- $\quad$ effect of future (predicted) disturbances $n(t+k \mid t)$.

The component $y_{\text {base }}(t+k \mid t)$ can be easily obtained using (6)(7)(8) taking $u_{\text {base }}(t+k \mid t)$ as the model input for (7).

$$
\begin{array}{llr}
\hline y_{\text {optimize }}(t+k \mid t): & & \\
\bullet \quad \text { effect of the optimizing future control actions } \\
& \left\{\delta u(t \mid t), \quad \delta u(t+1 \mid t), \ldots \delta u\left(t+N_{u}-1 \mid t\right)\right\} & \text { with } \\
& \delta u(t+k \mid t)=u(t+k \mid t)-u_{\text {base }}(t+k \mid t) .
\end{array}
$$

Fig. 2 depicts the concepts of base and optimizing controls. Notice that $u(t+k \mid t)$ is constrained to be constant from $k=N_{u}$ on (and this is realized by selecting $u_{\text {base }}(t+k \mid t)$ constant from $k=N_{u}$ on and by imposing that $\delta u(t+k \mid t)$ should be constant from $k=N_{u}$ on). The design parameter $N_{u}$ is called the control horizon, a well-known concept in MPC-literature.

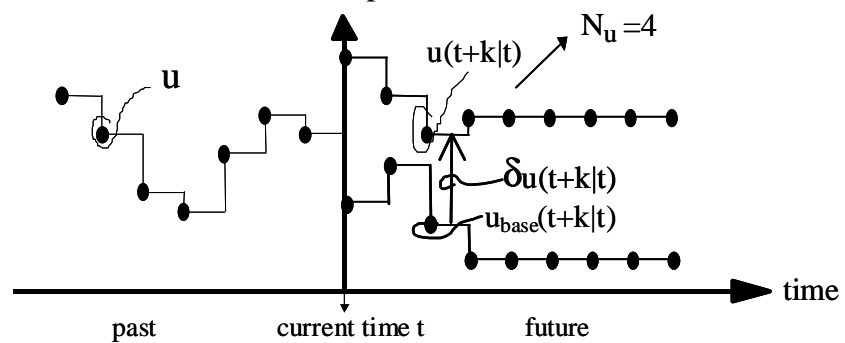

Fig. 2. The EPSAC concept of base/optimizing controls From Fig. 2 it is obvious that the component $y_{\text {optimize }}(t+k \mid t)$ is the cumulative effect of a series of impulse inputs and a step input: 
- $\quad$ an impulse with amplitude $\delta u(t \mid t)$ occurring at time $t$, resulting in a contribution $h_{k} \delta u(t \mid t)$ to the process output at time $t+k$ ( $k$ sampling periods later);

- $\quad$ an impulse with amplitude $\delta u(t+1 / t)$ occurring at time $t+1$, resulting in a contribution $h_{k-1} \delta u(t+1 / t)$ to the predicted process output at time $t+k(k-1$ sampling periods later);

- etc;

- finally a step $\delta u\left(t+N_{u}-1 \mid t\right)$ at time $t+N_{u}-1$, resulting in a contribution $g_{k-N_{u}+1} \delta u\left(t+N_{u}-1 \mid t\right)$ to the predicted process output at time $t+k$.

The cumulative effect of all impulses and the step is:

$$
\begin{aligned}
y_{\text {optimize }}(t+k \mid t) & =h_{k} \delta u(t \mid t)+h_{k-1} \delta u(t+1 \mid t)+ \\
& +\ldots+g_{k-N_{u}+1} \delta u\left(t+N_{u}-1 \mid t\right)
\end{aligned}
$$

The parameters $g_{1}, g_{2}, \ldots g_{k}, \ldots g_{N_{2}}$ are the coefficients of the unit step response of the system, i.e. the response of the system for a stepwise change of the input (with amplitude 1). The parameters $h_{1}, h_{2}, \ldots h_{k}, \ldots h_{N_{2}}$ are the coefficients of the unit impulse response of the system and can be easily calculated from the step response coefficients and vice versa: $h_{k}=g_{k}-g_{k-1}\left(\right.$ and $\left.h_{0}=h_{-1}=\ldots=g_{0}=g_{-1}=\ldots \equiv 0\right)$.

Using (9) and (10), the key EPSAC-MPC equation:

is obtained, where:

$$
\mathbf{Y}=\overline{\mathbf{Y}}+\mathbf{G U}
$$

$$
\begin{aligned}
\mathbf{Y} & =\left[y\left(t+N_{1} \mid t\right) \cdots y\left(t+N_{2} \mid t\right)\right]^{T} \\
\overline{\mathbf{Y}} & =\left[y_{\text {base }}\left(t+N_{1} \mid t\right) \cdots y_{\text {base }}\left(t+N_{2} \mid t\right)\right]^{T} \\
\mathbf{U} & =\left[\delta u(t \mid t) \cdots \delta u\left(t+N_{u}-1 \mid t\right)\right]^{T} \\
\mathbf{G} & =\left[\begin{array}{ccccc}
h_{N_{1}} & h_{N_{1}-1} & h_{N_{1}-2} & \cdots & g_{N_{1}-N_{u}+1} \\
h_{N_{1}+1} & h_{N_{1}} & h_{N_{1}-1} & \cdots & \cdots \\
\ldots & \cdots & \cdots & \cdots & \ldots \\
\ldots & \ldots & \cdots & \cdots & \ldots \\
h_{N_{2}} & h_{N_{2}-1} & h_{N_{2}-2} & \cdots & g_{N_{2}-N_{u}+1}
\end{array}\right]
\end{aligned}
$$

with the horizons $N_{1}, N_{2}$ being design parameters, $N_{1}$ being equal to the time-delay, in samples. The controller output is then the result of minimizing the cost function:

$$
J(\mathbf{U})=\sum_{k=N_{1}}^{N_{2}}[r(t+k \mid t)-y(t+k \mid t)]^{2}
$$

where $r(t+k / t)$ is the desired reference trajectory:

$$
r(t+k \mid t)=\alpha r(t+k-1 \mid t)+(1-\alpha) w(t+k \mid t)
$$

with $w$ the setpoint and $\alpha$ a tuning parameter $(0 \leq \alpha \leq 1)$ which specifies the desired closed-loop speed. The cost function (13) is a quadratic form in $\mathbf{U}$, having the following structure using the matrix notation from (12) and with $\mathbf{R}$ defined similarly to $\mathbf{Y}$ :

$$
J(\mathbf{U})=[\mathbf{R}-\overline{\mathbf{Y}}-\mathbf{G U}]^{-1}[\mathbf{R}-\overline{\mathbf{Y}}-\mathbf{G U}]
$$

which leads after minimization w.r.t. $\mathbf{U}$ to the optimal solution:

$$
\mathbf{U}^{*}=\left[\mathbf{G}^{T} \mathbf{G}\right]^{-1} \mathbf{G}^{T}(\mathbf{R}-\overline{\mathbf{Y}})
$$

The matrix $\mathbf{G}^{\mathbf{T}} \mathbf{G}$ which has to be inverted has dimension $N_{u} \mathbf{x}$ $N_{u}$. For the default case $N_{u}=1$, this results in a simple scalar control law. Only the first element $\delta u(t / t)$ in $\mathbf{U}^{*}$ is required in order to compute the actual control input applied to the process:

$$
u(t)=u_{\text {base }}(t \mid t)+\delta u(t \mid t)=u_{\text {base }}(t \mid t)+\mathbf{U}^{*}(1)
$$

At the next sampling instant $t+1$, the whole procedure is repeated taking into account the new measurement information $y(t+1)$. This is called the principle of receding horizon control, another well-known MPC-concept.

Equation (15) can be re-written as a quadratic form in $\mathrm{U}$ as:

$$
J(\mathbf{U})=\mathbf{U}^{\mathrm{T}} \mathbf{H} \mathbf{U}+\mathbf{2} \mathbf{f}^{\mathrm{T}} \mathbf{U}+\mathbf{c}
$$

where: $\quad \mathbf{H}=\left[\mathbf{G}^{T} \mathbf{G}\right], \quad \mathbf{f}=-\left[\mathbf{G}^{\mathrm{T}}(\mathbf{R}-\overline{\mathbf{Y}})\right], \mathbf{c}=(\mathbf{R}-\overline{\mathbf{Y}})^{\mathrm{T}}(\mathbf{R}-\overline{\mathbf{Y}})$. Minimization of $J(\mathbf{U})$ results in the solution:

$$
\mathbf{U}^{*}=-\mathbf{H}^{-1} \mathbf{f}
$$

which is equivalent to (16). It is worthwhile to notice that optimization with input constraints (on the manipulated variable) are accepted only if the control horizon $N_{u}>1$. Since in this study the control horizon is unitary, the input is constrained using clipping (De Keyser 2003).

\subsection{Nonlinear EPSAC}

The concept of base and optimizing controls/responses as introduced in formula (9) is theoretically only valid for linear systems, as it is based on the superposition principle. However, for nonlinear systems, by selecting the base control $u_{\text {base }}(t+k / t)$ appropriately, the $2^{\text {nd }}$ term in (9) can gradually be made equal to zero in an iterative way. This then results in the optimal solution, also for nonlinear systems, because the superposition principle is no longer involved. The procedure can be summarized as follows. At each sampling instant:

1) select an initial $\left\{u_{\text {base }}(t+k / t), k=0 \ldots N_{u}-1\right\}$; in the case of linear models, the choice is irrelevant for the solution, some simple examples being $u_{\text {base }}(t+k / t) \equiv 0$ or $u_{\text {base }}(t+k / t) \equiv u(t-1) \quad$ (the last one corresponds to the GPC approach). However, for nonlinear models, it is the objective to obtain finally (in an iterative way) a control policy $u_{\text {base }}(t+k / t)$, which is as close as possible to the optimal strategy $u(t+k / t)$ (thus bringing the optimizing control actions $\delta u(t+k / t)$ and the corresponding term 
$y_{\text {optimize }}(t+k / t)$ practically to zero). In order to minimize the number of iterations, it is thus wise to make a 'good' initial guess for $u_{\text {base }}(t+k / t)$. A simple but effective choice is to start with $u_{\text {base }}(t+k / t) \equiv u(t+k / t-1)$, which is the optimal control policy derived at the previous time sample.

2) once a $\left\{u_{\text {base }}(t+k / t), k=0 \ldots N_{u}-1\right\}$ has been chosen, calculate $\quad\left\{\delta u(t+k / t), k=0 \ldots N_{u}-1\right\}$ and the controls $\left\{u(t+k / t)=u_{\text {base }}(t+k / t)+\delta u(t+k / t), k=0 \ldots N_{u}-1\right\} \quad$ as explained for linear EPSAC. For a linear model, these are the optimal controls and the procedure can be stopped. For a nonlinear model, these are not the optimal controls because the principle of superposition does not hold, but it is reasonable to assume that the resulting $u(. /$.$) are 'closer' to$ the optimal controls than the previous guess $u_{\text {base }}(. /$.$) ; so, for a$ nonlinear model:

3 ) continue the procedure - at the same sampling instant! by taking these $\left\{u(t+k / t), k=0 \ldots N_{u}-1\right\}$ as a new $\left\{u_{\text {base }}(t+k / t), k=0 \ldots N_{u}-1\right\}$ and return to step 2).

Continuing this iteration and considering the above mentioned assumption to be true, it can be expected that $u_{\text {base }}(. /$.$) will converge to the optimal u(. /$.$) . Indeed, each time$ that $u_{\text {base }}(. /$.) is closer to $u(. /$.$) , it means that the \delta u(. /$.$) are$ smaller and thus also the term $y_{\text {optimize }}(. /$.$) in (11) becomes$ smaller. The superposition principle - used in (11), but in fact invalid for nonlinear systems - is step-by-step 'less involved' (it has less impact). Finally, when the $\delta u(. /$ ) are (practically) zero, the superposition principle is no longer involved and the calculated control signal will thus be optimal, also for the nonlinear system.

It is important to realize that in this specific Nonlinear MPC approach, the nonlinear system is never linearized. The nonlinear model is directly used to calculate in every iteration the base responses $y_{\text {base }}(. /$.$) as well as to calculate the required$ step/impulse-response coefficients $\left\{g_{\mathrm{i}}, h_{\mathrm{i}}\right\}$. These are the only values required in (16) or equivalently, in (19).

\section{RESULTS AND DISCUSSION}

Controller performance was evaluated using different parameter sets (Table 1) to simulate the patients. Parameter set 1 was used for the prediction model. The tuning of the NEPSAC parameters was un-changed. It was considered optimal for the following values: $N_{u}=1, N_{l}=1, N_{2}=N_{1+} 15$, $\alpha=0.9$. The iteration in the NEPSAC procedure was stopped either when the value of the $\delta u(. /$.$) was 2000$ times smaller than the one of the updated $u_{\text {base }}(. /$.$) , or when the number of$ iterations exceeded 30 . It is recommended that the controller updates the drug rate every 10 seconds, therefore a sample time of 10 seconds has been adopted.

Fig.3. shows the time course of muscle relaxation for reference tracking and disturbance rejection. The first scenario represents the ideal case, when there are no modelling errors. Of course this situation never appears in practice, but it helps us get a better view upon the EPSAC performance compared to the situation when it is applied to different patients. In the ideal case the reference is reached in about 6 minutes, while for the worst case scenario the time required is two times higher.

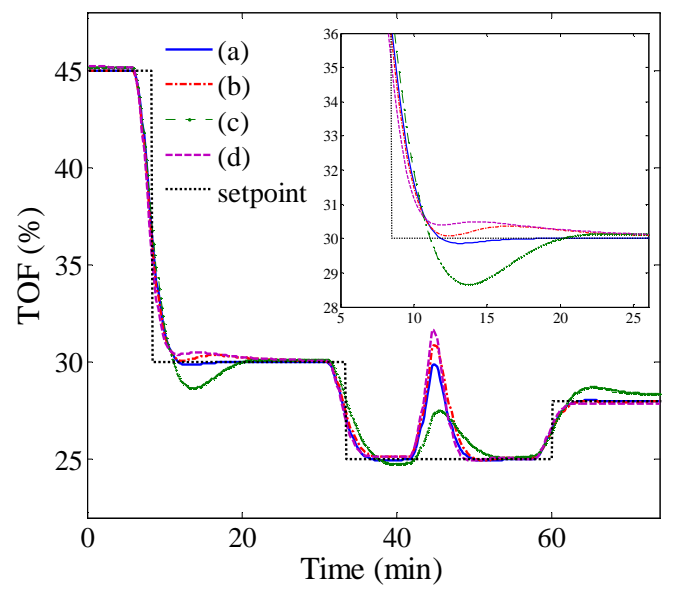

Fig.3. Closed-loop response for the controlled variable (TOF), using different parameter sets to simulate the patient: (a) set 1 ; (b) set 2; (c) set 3; (d) set 4

The muscle relaxation disturbance associated with an empty infusion bag was considered. Since transport of rocuronium into the patient is stopped, this disturbance is independent of the controller. When the infusion is restarted, a bolus of rocuronium is quickly administered to the patient, resulting in a transient decrease in muscle relaxation around minute 45 .

The respective control effort required to produce the above discussed performance is depicted in Fig 4. Since each patient has a different sensitivity to the drug, for the same reference value, the controller sends different drug rates. The patient simulated with the $3^{\text {rd }}$ set of parameters has a higher sensitivity to rocuronium, therefore less drug is required. The effect of the disturbance caused by the empty infusion bag is lower in this case.

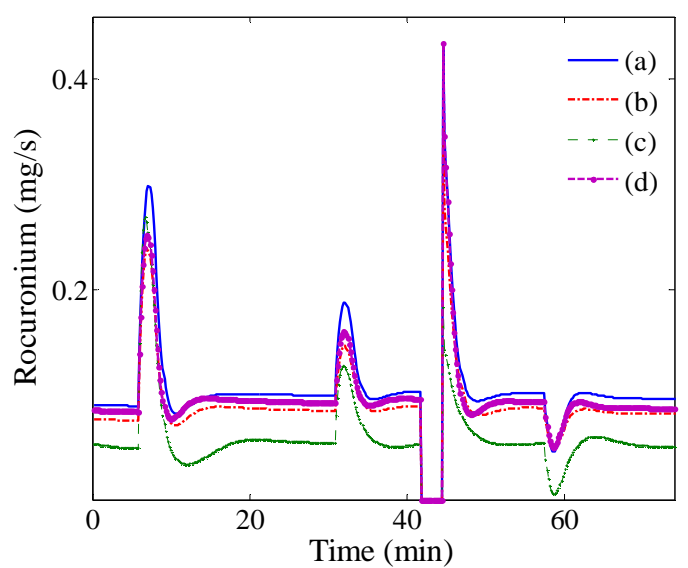

Fig.4. Closed-loop response for the manipulated variable (rocuronium), using different parameter sets to simulate the patient: (a) set 1; (b) set 2; (c) set 3; (d) set 4

An overview of the number of iterations required in the NEPSAC procedure, as explained in section 3.3, is given in Fig 5. All four scenarios find the optimal $\delta u(. /$.) values within the maximum allowed number of iterations. It can be 
observed that less iterations are required when using the $3^{\text {rd }}$ set of parameters, while the higher number of iterations is needed for case (a).

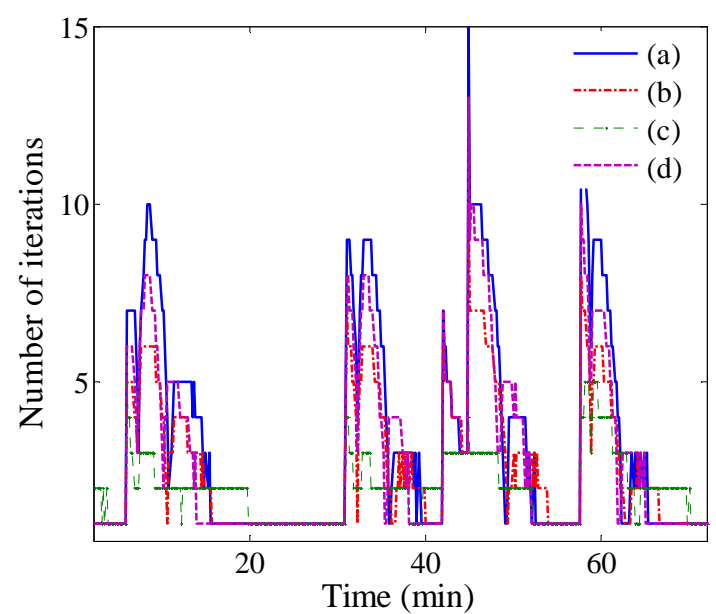

Fig.5. Number of iterations required by the controller to find the optimal control signal, using different parameter sets to simulate the patient: (a) set 1; (b) set 2; (c) set 3; (d) set 4

Although the controller presented is non-adaptive, it performs sufficiently well in this first stage, on several simulated patients. Further investigation is required to select the proper prediction model and more robustness tests are necessary before applying this controller in routine clinical conditions.

\section{CONCLUSIONS}

This contribution presents EPSAC control strategy applied to the administration of rocuronium in general anaesthesia. An overview of a PK-PD model is given and several parameter sets are used to simulate different patients. Performance assessment shows good results in set-point tracking and disturbance rejection. The controller proved insensitivity to inter-patient variability and disturbance represented by an unnoticed empty infusion bag that can occur in clinical conditions. The strategy can be easily extended to a multipleinput multiple-output system, taking into account several drugs administered during general anaesthesia.

\section{ACKNOWLEDGEMENT}

R. Hodrea is supported by the project "Improvement of the doctoral studies quality in engineering science for development of the knowledge based society-QDOC" contract no. POSDRU/107/1.5/S/78534, project co-funded by the European Social Fund through the Sectorial Operational Program Human Resources 2007-2013. C.M. Ionescu is a post-doctoral fellow of Flanders Research Foundation.

\section{REFERENCES}

Alvarez-Gomez JA, Estelles ME, Fabregat J, Perez F, Brugger AJ. (1994). Pharmacokinetics and pharmacodynamics of rocuronium bromide in adult patients. Eur J Anaesthesiol; 11 (Suppl 9): 53 \pm 6

Cooper RA, Maddenini VR, Mirakhur RK, Wierda JMKH, Brady M, Fitzpatrick KTJ. (1993) Time course of neuromuscular effects and pharmacokinetics of rocuronium bromide (Org 9426) during isoflurane anaesthesia in patients with and without renal failure. $\mathrm{Br}$ J Anaesth; 71: 222 \pm 6

De Keyser, R .(2003). Model Based Predictive Control, Invited Chapter in UNESCO Encyclopedia of Life Support Systems (EoLSS), article 6.43.16.1, Eolss Publishers Co Ltd, Oxford 83, ISBN 09542989 18-2634.

De Keyser R., Ionescu C. (2003) The Disturbance Model in Model Based Predictive Control, IEEE Conference on Control Applications (CCA'2003), Istanbul, Turkey, CD paper CF-001472

De Vries JW, Ros HH, Booij LHDJ. (1986). Infusion of vecuronium controlled by a closed loop system. $\mathrm{Br} J$ Anaesth;58:100-3

Farenc C., Lefrant J.Y., Audran M., Bresolle F. (2001). Pharmacokinetic-Pharmacodynamic Modelling of Atracurium in Intensive Care Patients, J Clin Pharmacol, 41-44

Foldes FF., Nagashima H., Nguyen HD., Schiller WS., Mason M., Ohata Y. (1991). The neuromuscular effects of ORG 9426 in patients receiving balanced anaesthesia. Anesthesiology; 75:191-196

Kansanaho M, Olkkola KT. (1996). Performance assessment of an adaptive model-based feedback controller: Comparison between atracurium mivacurium, rocuronium and vecuronium. J Clin Monit Comput (ITE); 134:217-24

McCoy EP, Mirakhur RK, Maddineni VR, Wierda JMKH, Proost JH. (1996). Pharmacokinetics of rocuronium after bolus and continuous infusion during halothane anaesthesia. Br J Anaesth; 76: 29+33

Sheiner LB, Stanski DR, Vozeh S, Miller RD, Ham J. Simultaneous modeling of pharmacokinetics and pharmacodynamics: application to D-tubocurarine. Clin Pharmacol Ther (1979); 25: 358 \pm 71

Szenohradszky J, Fisher DM, Segredo V, et al. (1992) Pharmacokinetics of rocuronium (ORG 9426) in patients with normal renal function or patients undergoing renal transplantation. Anesthesiology; 77: 899 \pm 904

Vermeyen KM., Hoffman VL., Saldien V. (2003). Target controlled infusion of rocuronium:analysis of effect data to select a pharmacokinetic model. $\mathrm{Br} J$ Anaesth 90(2):183-8

Wierda JMKH, Kleef UW, Lambalk LM, Kloppenburg WD, Agoston S. (1991) The pharmacodynamics of Org 9426, a new nondepolarising neuromuscular blocking agent, in patients anaesthetized with nitrous oxide, halothane and fentanyl. Can J Anaesth; 38: 430 \pm 5

Wierda JMKH, Kleef UW, Lambalk LM, Kloppenburg WD, Morgan, G. E., and Mikhail, M. S. (1996). Clinical Anesthesiology, second ed. Prentic-Hall International, Inc 